\title{
Influence of MnTE-2-PyP on Inflammation and Lipid Peroxidation in Mouse Asthma Model
}

\author{
Lyudmil Terziev', Veneta Shopova², Violeta Dancheva², Galya Stavreva ${ }^{3}$, Milena Atanasova ${ }^{4}$, \\ Angelina Stoyanova ${ }^{5}$, Tzvetan Lukanov ${ }^{1}$, Anelia Dimitrova ${ }^{6}$ \\ ${ }^{1}$ Sector of Clinical Immunology and Allergology, Medical University, Pleven, Bulgaria \\ ${ }^{2}$ Sector of Disaster Medicine, Medical University, Pleven, Bulgaria \\ ${ }^{3}$ Sector of Experimental and Clinical Pharmacology, Medical University, Pleven, Bulgaria \\ ${ }^{4}$ Sector of Biology, Medical University, Pleven, Bulgaria \\ ${ }^{5}$ Sector of Chemistry, Medical University, Pleven, Bulgaria \\ ${ }^{6}$ Sector of Pathophysiology, Medical University, Pleven, Bulgaria \\ Email: luterzi@mail.bg
}

Received January 13, 2012; revised March 6, 2012; accepted March 15, 2012

\begin{abstract}
Our aim was to investigate the effects of MnTE-2-PyP on some markers of inflammation and lipid peroxidation in mouse asthma model. 24 female mice were divided into four groups: group 1, controls; group 2, injected with ovalbumin (OVA); group 3, treated with MnTE-2-PyP; and group 4, treated with ovalbumin and MnTE-2-PyP. The mice from groups 2 and 4 were injected with $10 \mu \mathrm{g}$ OVA and $1 \mathrm{mg}$ Imject Alum ${ }^{\circledR}$ in $100 \mu \mathrm{L}$ phosphate buffered saline (PBS) on days 0 and 14. The animals from groups 1 and 3 were injected with $100 \mu \mathrm{L}$ PBS + Imject Alum ${ }^{\circledR}(1: 1)$. The animals from groups 2 and 4 were subjected to a 30 min aerosol challenge of $1 \%$ ovalbumin on days 24,25 and 26 and those from groups 1 and 3 were subjected to aerosol challenge of PBS at the same time and duration. One hour before inhalation, and 12 hours later the animals from groups 3 and 4 were injected with $100 \mu \mathrm{L}$ MnTE-2-PyP solution in PBS containing $5 \mathrm{mg} / \mathrm{kg}$. The total cell number, total protein content and 8-isoprostane, IL-4 and IL-5 levels in the bronchialveolar lavage fluid increased in group 2 as compared to the control group. Malone dialdehyde content in the lung homogenate and IgE levels in the serum also increased in this group. The total cell number, total protein content, and levels of 8-isoprostane, IL-4, IL-5 and IgE decreased significantly in group 4 as compared to the OVA group. The parameters set out above in group 3 did not differ significantly from those of the control group. MnTE-2-PyP administered intraperitoneally, 48 hours after the last nebulization, reduced the inflammation and lipid peroxidation in mouse asthma model.
\end{abstract}

Keywords: Asthma; Inflammation; Interleukins; 8-Isoprostane; Lipid Peroxidation; MnTE-2-PyP

\section{Introduction}

Asthma is a lung disease characterized by airspace inflammation and oxidative stress [1-4]. Elevated levels of reactive oxygen species (ROS), released by inflammatory cells, either directly or through the formation of products of lipid peroxidation, play a role in enhancing the inflammatory response in these diseases. The presence of oxidative stress is important in the pathogenesis, severity and treatment of asthma [5]. Increasing evidence suggests that abnormalities in mitochondria are involved in several mitochondrial diseases, but also in the development of asthma [6,7]. Recently, antioxidants to prevent and to treat mitochondria in patients with mitochondrial diseases, including asthma, has received much attention, especially because antioxidant approaches seem to have few or no adverse effects [8]. Different classes of antioxidants are known. Among them, the group of catalytic manganese metalloporphyrins takes center stage with their accumulation into mitochondria. They have at least four antioxidant properties, such as removal of superoxide $\left(\mathrm{O}_{2}^{-}\right)$, hydrogen peroxide $\left(\mathrm{H}_{2} \mathrm{O}_{2}\right)$, peroxynitrite $\left(\mathrm{ONOO}^{-}\right)$, and lipoperoxides $[9,10]$. Based on this information, we set the goal to investigate the effects of MnTE-2-PyP (Manganese(III) 5, 10, 15, 20-tetrakis ( $N$ ethylpyridinium-2-yl)porphyrin), a manganese-mesoporphyrin also known as AEOL-10113, on markers of inflammation and lipid peroxidation in a mouse ovalbumin (OVA) sensitization model of asthma [11].

\section{Materials and Methods}

\subsection{Chemicals}

Ovalbumin, grade $\mathrm{V}$, and phosphate buffered saline (PBS), were purchased from Sigma-Aldrich Company, Nitrocellulose filters with $5 \mu \mathrm{m}$ pores were from Milli- 
pore Corp, IL-4 and 5 ELISA Kits were from R\&D Systems, 8-Isoprostane EIA Kit was from Cayman chemicals, Mouse IgE ELISA Sets were purchased from BD Biosciences, and the Imject Alum ${ }^{\mathbb{B}}$ was from the Pierce Chemical Company (USA). MnTE-2-PyP was kindly provided by Prof. Ines Batinić-Haberle from the Department of Radiation Oncology, Duke University Medical Center, Durham, North Carolina, USA.

\subsection{Animals and Experimental Protocol}

The experiment was performed in accordance with Animal Welfare Regulations and was approved by the University Ethics Committee.

The study was carried out on 24 female C57B1/6 mice (weight $20 \pm 2 \mathrm{~g}, 8$ - 10 week old). The animals were raised at the University vivarium at a temperature of $22^{\circ} \mathrm{C}$ $\pm 2^{\circ} \mathrm{C}$ and humidity of $50 \% \pm 10 \%$, and were given a normal pelleted diet and water ad libitum. The mice were divided into four groups: group1, controls; group 2, injected with ovalbumin; group 3, treated with MnTE-2PyP and group 4, treated with OVA and MnTE-2-PyP. Airway inflammation was induced by OVA immunization and challenge. The animals from groups 1 and 3 were injected i.p. with a $100 \mu \mathrm{L}$ phosphate-buffed saline (PBS) + Imject AlumR (1:1) on days 0 and 14. The animals from groups 2 and 4 were injected with a $100 \mu \mathrm{L}$ ovalbumin solution, containing $20 \mu \mathrm{g}$ OVA on the same days. On days 24,25 and 26, mice from groups 1 and 3 were given inhalation with PBS for $30 \mathrm{~min}$, and those from groups 2 and 4 were given inhalation with $1 \%$ ovalbumin solution (OVA dissolved in PBS). For this purpose, a special plexiglass chamber was used. One hour before inhalation, and 12 hours later the animals from groups 1 and 2 were injected i.p. with $100 \mu \mathrm{L}$ PBS, and those from groups 3 and 4 received a $100 \mu \mathrm{L}$ MnTE-2-Pyp dissolved in PBS, containing $5 \mathrm{mg} / \mathrm{kg}$, that is, the total daily dose was 10 $\mathrm{mg} / \mathrm{kg}$.

The solution was sterilized by filtration through 0.2 $\mu \mathrm{m}$ filters.

\subsection{Bronchoalveolar Lavage Fluid (BALF)}

To obtain BALF, the animals were sacrificed on day 28 (48 hours after the last inhalation) under thiopental anaesthesia $(50 \mathrm{mg} / \mathrm{kg})$. The chest was opened and the lungs were perfused in situ via the right heart ventricle with saline $(10 \mathrm{~mL})$. Triple lavage of the left lung through the trachea was performed with a total volume of $2.5 \mathrm{~mL}$ of saline. The right lung was ligated at the hilum, cut and then removed from the chest and used to prepare the lung homogenate.

\section{Cytological, Biochemical and Immunological Assays of BALF}

One aliquot of the BALF was used for the purpose of total cell number $\times 10^{5} / \mathrm{mL}$. The cells were then removed by centrifugation at $300 \times \mathrm{g}$ for $10 \mathrm{~min}$. The supernatant of BALF was used to measure interleukins and 8-isoprostane levels. The cell pellet was resuspended in 0.5 $\mathrm{mL}$ of saline, and differential cell count using Millipore filters by the method of Danos and Keebler, modified by Saltini [12] was performed. The total protein content in $\mathrm{ng} / \mathrm{mL}$ by the method of Lowry et al. [13], the levels of IL-4 and IL-5 in $\mathrm{pg} / \mathrm{mL}$ by the ELISA method, and the level of 8-isoprostane in $\mathrm{ng} / \mathrm{mL}$ by the ELISA method in accordance with manufacturer's instructions, were investigated in the supernatant of BALF.

\subsection{Biochemical Assays of Lung Homogenate}

Lung homogenate was obtained from the right lung. The tissue was homogenized with potassium chloride $(\mathrm{KCl})$ in 1:10 ratio (lung mass by $\mathrm{KCl}$ solution volume). The homogenate was centrifuged $(9000 \times \mathrm{g}, 30 \mathrm{~min})$, and the supernatant was stored on ice. Malone dialdehyde (MDA) content in nmol/g was measured by the method of Ohkawa et al. [14].

\subsection{Immunological Assay of Serum}

Blood was drown from the abdominal aorta by using vacuum blood collection tubes. The blood was allowed to clot for 30 minutes, and then centrifuged at $1000 \times \mathrm{g}$ for $10 \mathrm{~min}$ to achieve serum separation. The samples were kept frozen at $-20^{\circ} \mathrm{C}$ until serum $\mathrm{IgE}$ analysis in $\mathrm{ng} / \mathrm{mL}$ was made by the ELISA method in accordance with manufacturer's instructions.

\subsection{Statistical Analysis}

Experimental data were analyzed using SPSS 14 . When we tested for normality, one variable-MDA showed nonparametric distribution, and we used medians, interquartile range and Mann-Whitney test for comparison. For the rest of the variable we applied post-hoc ANOVA test and data were presented as mean \pm standart error of mean (SEM). $P<0.05$ was considered statistically significant.

\section{Results}

The total cell number in group 2 (OVA-sensitized mice) increased more than four fold in BALF $(479 \%$ as compared to the controls, $P=0.025$ ). The increase of this parameter in group 4 (OVA + MnTE-2-PyP) was significantly lower $(178 \%)$ than that in group 2 . The eosinophil percentage was $28 \%$ in group 2 and $18 \%$ in group 4 , versus $0.5 \%$ in the control group (Table 1). The total protein content in group 2 showed the same dynamics (Table 1). The levels of IL-4 and IL-5 increased sharply in group 2 (OVA) up to $1849 \%(P=0.0006)$ and $350 \%$ $(P=0.016)$ respectively, in comparison with the control 
Table 1. Effect of MnTE-2-PyP on markers of inflammation in BALF and lipid peroxidation in lung homogenate in mouse model of asthma.

\begin{tabular}{|c|c|c|c|c|}
\hline \multicolumn{5}{|c|}{28 day after treatment (48 hours after the last inhalation) } \\
\hline Parameters & Control & OVA & MnTE-2-PyP & OVA + MnTE-2-PyP \\
\hline Total cell number in BALF $\left(\times 10^{5} \mathrm{~mL}\right)$ & $2.26 \pm 0.09$ & $10.84 \pm 2.34^{*}$ & $4.00 \pm 0.40$ & $4.04 \pm 0.41^{\dagger}$ \\
\hline AMas (\%) & 90.2 & 58.8 & 85.3 & 70.0 \\
\hline PMN (\%) & 4.6 & 8.2 & 5.5 & 5.8 \\
\hline Eo (\%) & 0.5 & 28.0 & 2.5 & 18.0 \\
\hline Total protein content $(\mathrm{mg} / \mathrm{mL})$ mean \pm SEM & $0.454 \pm 0.025$ & $0.563 \pm 0.023^{*}$ & $0.419 \pm 0.038$ & $0.463 \pm 0.050^{\dagger}$ \\
\hline MDA content $(n \mathrm{~mol} / \mathrm{g})$ Median & 22.86 & $42.7^{*}$ & 22.68 & $20.86^{\dagger}$ \\
\hline Min - max & $21.56-26.88$ & $39.2-46.20$ & $15.12-32.48$ & $19.04-22.68$ \\
\hline $\mathbf{Q}_{3}-\mathbf{Q}_{1}$ & 2.66 & 6.8 & 17.36 & 3.64 \\
\hline
\end{tabular}

Abbreviations: OVA, ovalbumin; MnTE-2-PyP, Manganese(III) 5, 10, 15, 20-tetrakis(Nethylpyridinium-2-yl)porphyrin; AMas, alveolar macrophages; PMN, polymorphonuclear leukocytes; Eo, eosinophils; IL-4 and IL-5, interleukins 4 and 5; IgE, immunoglobulin E; MDA, malone dialdehyde; SEM, standart error of mean; $\mathrm{Q}_{3}-\mathrm{Q}_{1}$, interquartile range. ${ }^{*}:$ Different from control at $P<0.05 ;{ }^{\dagger}$ : Different from group $2(\mathrm{OVA})$ at $P<0.05$.

group (Figures 1 and 2). OVA and antioxidant treatment (group 4) decreased significantly these parameters as compared to group 2 (OVA), up to $115 \%(P=0.00003)$ and $123 \%(P=0.007)$ respectively. The IgE levels in the group treated with OVA alone elevated up to $340 \%$ as compared to the control animals $(P=0.00008)$. The increase in group 4 (OVA + MnTE-2-PyP) was significantly lower $(264 \%, P=0.049)$ as compared to group 2 (Figure 3). The changes in 8-isoprostane levels in BALF and MDA content in lung homogenate, which are markers of lipid peroxidation, followed the same trend among the four groups. The level of 8-isoprostane in group 2 was three times higher than that in the control group $(P=$ 0.03 ), whereas in group 4 (treated with OVA and MnTE2-PyP) it was significantly lower than that in group 2, and the values were approximate to those of the controls (Figure 4). The MDA content increased up to $187 \%$ in group 2 as compared to the control group. In group 4 this content was approximate to that in the controls, and was lower as compared to group $2(P=0.03)$, (Table 1). The levels of interleukins and IgE as well as 8-isoprostane in group 3 (MnTE-2-PyP alone) did not differ significantly from those of control group.

\section{Discussion}

The experimental data of our study showed that the MnTE-2-PyP has a beneficial effect on the indicators of inflammation and lipid peroxidation. Metaloporphyrins are a new and potent class of lipid peroxidation inhibitors. Their potency is connected not only to their redox potential, but also to other factors that can contribute to their ability to act as electron acceptors. MnTE-2-PyP is very effective in the elimination of reactive species, particularly $\mathrm{O}_{2}^{-}$and $\mathrm{ONOO}^{-}$, with the highest rate constant among the other synthetic antioxidants [15-18]. Manganese(III) 5, 10, 15, 20-tetrakis( $N$-ethylpyridinium-2-yl) porphyrin (MnTE-2-PyP) and Manganese(III) 5, 10, 15, 20-tetrakis $(N$-hexylpyridinium-2-yl)porphyrin (MnTnHex2-PyP) have an excellent SOD activity in vitro and in vivo $[19,20]$. The biological role of MnTBAP and MnTE-2-PyP is related to their peroxynitrite-binding activity and reduction of the carbonate radical. Logcat

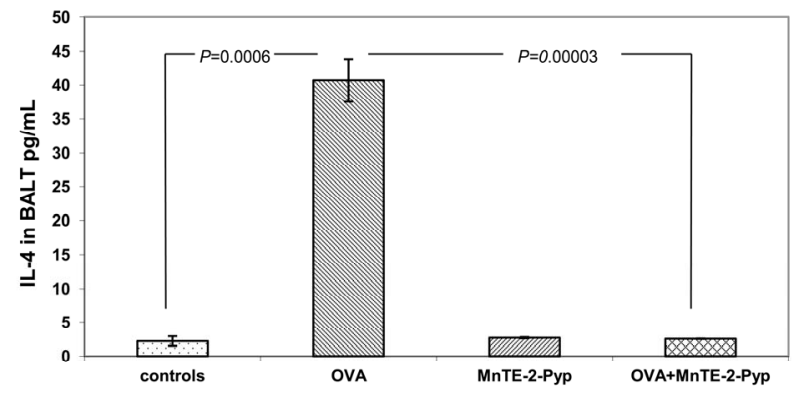

Figure 1. The level of IL-4 in BALF. Each point represents the mean \pm SEM for six mice.

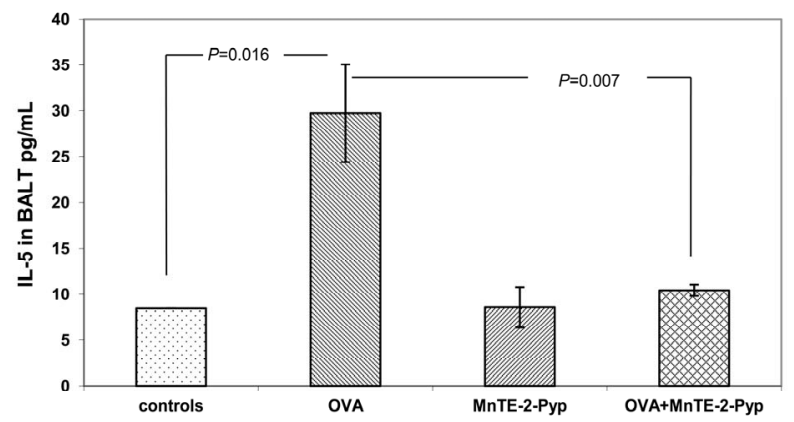

Figure 2. The level of IL-5 in BALF. Each point represents the mean \pm SEM for six mice. 


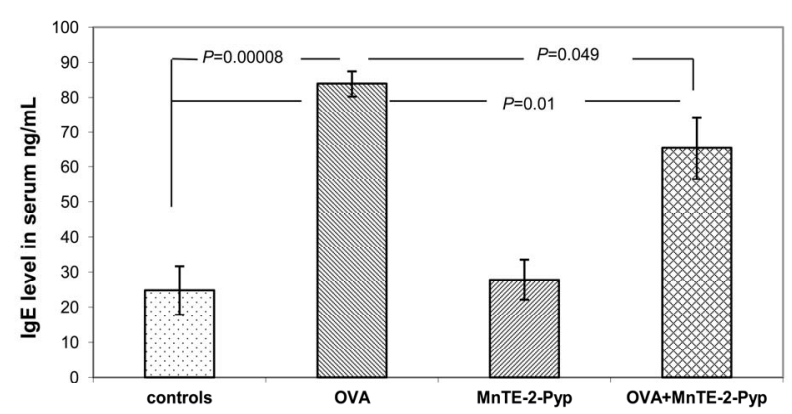

Figure 3. The level of IgE in serum. Each point represents the mean \pm SEM for six mice.

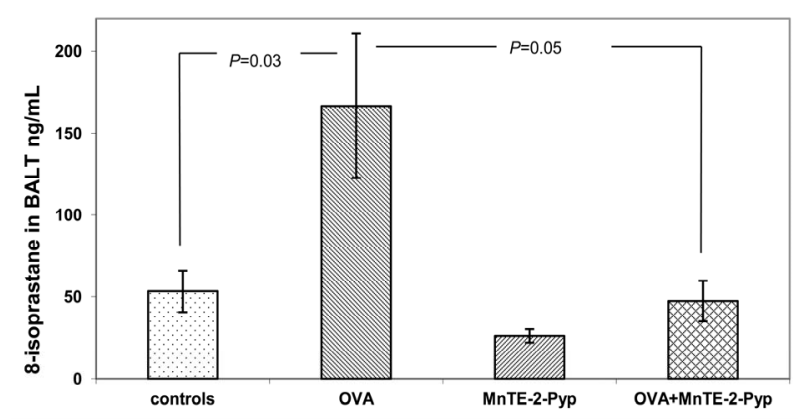

Figure 4. The level of 8-isoprostane in BALF. Each point represents the mean \pm SEM for six mice.

$\left(\mathrm{O}_{2}^{-}\right)$of MnTBAP is about 3.16, which is about $5-6$ times less-than the SOD activity of the powerful SOD mimetic MnTE-2-PyP and CuZn SOD. Positively charged MnTE-2-PyP and related analogues are very suitable for SOD mimetics and $\mathrm{ONOO}^{-} / \mathrm{CO}_{3}^{-}$cleaners. MnTE-2PyP has a potent catalytic antioxidant-like effect of extracellular superoxide dismutase. The strong porphyrinbased compounds are based on a "structure- activity" relationship, such as $\mathrm{Mn}$ (III) meso-tetreakis ( $N$-ethylpyridinium-2-yl)porphyrin (MnTE-2-PyP) and its hexyl analogue (MnTnHex-2-PyP) [21].

We applied antioxidants in a dose of $10 \mathrm{mg} / \mathrm{kg} /$ daily, divided into doses over 12 hours, starting with the assertion that good tolerance in mice is observed at two doses of $15 \mathrm{mg} / \mathrm{kg} /$ day and the plasma half-life varies considerably. This is the most commonly used (as single or multiple) therapeutic dose of this porphyrin [22]. MnTE2-PyP administered i.p. or orally reaches maximum concentration in plasma in 0.33 hours [16,17]. It accumulates at high levels in liver, kidney, and spleen, at moderate levels in lungs and heart, and at low levels in brain. The plasma half-life for a single i.p. dose of $10 \mathrm{mg} / \mathrm{kg}$ in mice is about 1 hour and the half-life in the body is significantly longer ranging from $60-135$ hours [22-24]. Studies reveal that MnTBAP and MnTM-4-PyP are effective in animal models of oxidative stress by forming superoxide [25-27], hydrogen peroxide [28,29] and peroxinitrite [30,31]. Many water-soluble meso substituted man- ganese porphyrins have a molecular weight above 800 , quickly pass through cell membranes and are distributed in mitochondria [23]. MnTE-2-PyP in the liver cells showed slightly higher accumulation in the mitochondria, as compared with the cytosol (Spasojevich I., unpublished data).

This compound not only decreases the primary insult of reactive species to biological molecules, but also inhibits the activation of transcription factors which in turn leads to suppression of expression of those cytokines and enzymes that perpetuate secondary oxidative stress [20,32]. According to Gauter-Fleckenstein et al. [33] the inhabitation of excessive cellular activity takes place through the suppression of the transcriptional activity, particularly suppressing HIF-1 $\alpha$ (hypoxia inducible factor $1 \alpha$ ) activetion in a long-lasting effect. Therefore reducing the total number of cells, the amount of total protein observed after treatment with mangan porphyrin can be explained by the ability of the antioxidants to inhibit the expression of VCAM-1 (vascular cell adhesion molecule-1) responsible for the accumulation of inflammatory cells, and thus to decrease airway hyperreactivity [34]. After intratracheal introduction, the antioxidant dramatically reduces the severity of airway inflammation in the airways in OVA-induced murine asthma. The reduction in the number of eosinophils, neutrophils and lymphocytes in BALF is more than $80 \%$ [34-36]. VCAM-1 and ICAM-1 (intra-cellular adhesion molecule) participate in the migration of eosinophils and neutrophils and contribute to eosinophilic inflammation in animal models. MnTE-2PyP has been shown to alter cell signaling and reduce inflammation by reducing NF-kB activity [37]. Piganelli et al. showed that MnTE-2-PyP inhibits T cell proliferation, while lipopolysaccha-ride-stimulated macrophages treated with the compound, inhibit TNF- $\alpha$ (tumor necrosis factor- $\alpha$ ) and NADPH release of superoxide [38]. The beneficial effects of $\mathrm{Mn}$ alkylpyri-dylporphyrins have been observed in various diseases, associated with oxidative stress, such as radiation injury, Alzheimer's disease, cancer, diabetes, central nervous system injuries, ischemia/reperfusion conditions, pulmonary emphysema, and other diseases [19,33,38-43].

\section{Acknowledgements}

This study was carried out with the financial support of Medical University-Pleven through the University Grants Commission (Project N 17/2009).

\section{REFERENCES}

[1] P. Kirkham and I. Rahman, "Oxidative Stress in Asthma and COPD: Antioxidants as a Therapeutic Strategy," Pharmacology and Therapeutics, Vol. 111, No. 2, 2006, pp. 476-494. doi:10.1016/j.pharmthera.2005.10.015 
[2] J. Bousquet, P. K. Jeffery, W. W. Busse, M. Johnson and A. M. Vignola, "Asthma. From Bronchoconstriction to Airways Inflammation and Remodeling," American Journal of Respiratory and Critical Care Medicine, Vol. 161, No. 5, 2000, pp. 1720-1745.

[3] R. Dworski, "Oxidant Stress in Asthma," Thorax, Vol. 55, No. 2, 2000, pp. S51-S53. doi:10.1136/thorax.55.suppl 2.S51

[4] P. K. Jeffery, "Comparison of the Structural and Inflammatory Features of Chronic Obstructive Pulmonary Disease and Asthma Giles F. Filley Lecture," Chest, Vol. 117.5 , No. 1, 2000, pp. 251S-260S. doi:10.1378/chest.117.5 suppl_1.251S

[5] J. Ciencewicki, S. Trivedi and S. R. Kleenberger, "Oxidants and the Pathogenesis of Lung Diseases," The Journal of Allergy and Clinical Immunology, Vol. 122, No. 3, 2008, pp. 456-470. doi:10.1016/j.jaci.2008.08.004

[6] B. A. Raby, B. Klanderman, A. Murphy, S. Mazza, C. A. Camargo Jr., E. K. Silverman and S. T. Weiss, "A Common Mitochondrial Haplogroup Is Associated with Elevated Total Serum IgE Levels," The Journal of Allergy and Clinical Immunology, Vol. 120, No. 2, 2007, pp. 351358. doi:10.1016/j.jaci.2007.05.029

[7] U. Mabalirajan, A. K. Dinda, S. Kumar, R. Roshan, P. Gupta, S. K. Sharma and B. Ghosh, "Mitochondrial Structural Changes and Dysfunction Are Associated with Experimental Allergic Asthma," Journal of Immunology, Vol. 181, No. 5, 2008, pp. 3540-3548.

[8] P. H. Reddy, "Mitochondrial Dysfunction and Oxidative Stress in Asthma: Implications for Mitochondria-Targeted Antioxidant Therapeutics," Pharmaceuticals, Vol. 4, No. 3, 2011, pp. 429-456. doi:10.3390/ph4030429

[9] M. Patel and B. J. Day, "Metalloporphyrin Class of Therapeutic Catalytic Antioxidants," Trends in Pharmacological Sciences, Vol. 2, No. 9, 1999, pp. 359-364. doi:10.1016/S0165-6147(99)01336-X

[10] B. J. Day, "Catalytic Antioxidants: A Radical Approach to New Therapeutics," Drug Discovery Today, Vol. 9, No. 13, 2004, pp. 557-566. doi:10.1016/S1359-6446(04)03139-3

[11] A. T. Nials and S. Uddin, "Mouse Models of Allergic Asthma: Acute and Chromic Allergen Challenge," Dis Models and Mechanisms, Vol. 1, No. 4-5, 2008, pp. 213220. doi: $10.1242 / \mathrm{dmm} .000323$

[12] C. Saltini, A. J. Hance, V. J. Ferrance, F. Basset, P. B, Bitterman and R. G. Crystal, "Acurate Quantification of Cells Received by Bronchoalveolar Lavage," The American Review of Respiratory Disease, Vol. 130, No. 4, 1984, pp. 650-658.

[13] O. H. Lowry, N. J. Rosenhbrough, A. L. Farr and R. J. Randall, "Protein Measurement with the Folin Phenol Reagent," The Journal of Biological Chemistry, Vol. 193, No. 1, 1951, pp. 265-275.

[14] H. Ohkawa, N. Ohishi and K. Yagi, "Assay for Lipid Peroxides in Animal Tissues by Thiobarbituric Acid Reaction," Analytical Biochemistry, Vol. 95, No. 2, 1979, pp. 351-358. doi:10.1016/0003-2697(79)90738-3

[15] G. DeFreitas-Silva, J. S. Rebouçças, I. Spasojević, L.
Benov, Y. M. Idemori and I. Batinić-Haberle, "SOD-Like Activity of $\mathrm{Mn}(\mathrm{II})$ beta-octabromo-meso-tetrakis(N-methylpyridinium-3-yl)porphyrin Equals That of the Enzyme Itself," Archives of Biochemistry and Biophysics, Vol. 477, No. 1, 2008, pp. 105-112. doi:10.1016/j.abb.2008.04.032

[16] I. Batinić-Haberle, I. Spasojevich, R. D. Stevens, P. Hambrigh and I. Fridovich, "Manganese(III)meso-tetrakis(ortho$\mathrm{N}$-alkylpyridyl)porphyrins. Synthesis, Characterization and Catalysis of $\mathrm{O}^{2-}$ Dismutation," Journal of the Chemical Society Dalton Transactions, 2002, No. 13, pp. 2689-2696.

[17] I. Batinić-Haberle, I. Spasojevich, R. D. Stevens, P. Hambright, P. Neta, A. Okado-Matsumoto and I. Fridovich, "New Class of Potent Catalysts of $\mathrm{O}^{2-}$ Dismutation. $\mathrm{Mn}$ (III)ortho-methoxyethylpyridyl and di-ortho-methoxyethylimidazolylporphyrins," Dalton Transactions, Vol. 11, 2004, pp. 1696-1702.

[18] J. S. Rebouças, I. Spasojević, D. H. Tjahjono, A. Richaud, F. Méndez, L. Benov and I. Batinić-Haberle, "Redox Modulation of Oxidative Stress by Mn Porphyrin-Based Therapeutics: The Effect of Charge Distribution," Dalton Transactions, Vol. 9, 2008, pp. 1233-1242.

[19] H. Saba, I. Batinić-Haberle, S. Munusamy, T. Mitchell, C. Lichti, J. Megyesi and L. A. MacMillan-Crow, "Manganese Porphyrin Reduces Renal Injury and Mitochondrial Damage during Ischemia/Reperfusion," Free Radical Biology and Medicine, Vol. 42, No. 10, 2007, pp. 15711578. doi:10.1016/j.freeradbiomed.2007.02.016

[20] Y. Zhao, L. Chaiswing, J. M. Velez, I. Batinić-Haberle, N. H. Colburn, T. D. Oberley and D. K. St. Clair, "p53 Translocation to Mitochondria Precedes Its Nuclear Translocation and Targets Mitochondrial Oxidative Defense Protein-Manganese Superoxide Dismutase," Cancer Research, Vol. 65, No. 9, 2005, pp. 3745-3750. doi:10.1158/0008-5472.CAN-04-3835

[21] I. Batinić-Haberle, S. Cuzzocrea, J. Rebouças, G. FerrerSueta; E. Mazzon, R. Di Paola, R. Radi, I. Spasojević, L. Benov and D. Salvemini, "Pure MnTBAP Selectively Scavenges Peroxynitrite over Superoxide: Comparison of Pure and Commercial MnTBAP Samples to MnTE-2-PyP in Two Models of Oxidative Stress Injury, an SOD-Specific Escherichia coli Model and Carrageenan-Induced Pleurisy," Free Radical Biology and Medicine, Vol. 46, No. 2, 2009, pp. 192-201.

doi:10.1016/j.freeradbiomed.2008.09.042

[22] I. Batinić-Haberle, J. S. Rebouças and I. Spasojević, "Superoxide Dismutase Mimics: Chemistry, Pharmacology, and Therapeutic Potencial," Antioxidants and Redox Signaling, Vol. 13, No. 6, 2010, pp. 877-918. doi:10.1089/ars.2009.2876

[23] I. Spasojevic, C. Yumin, T. Noel, Y. Yu, M. P. Cole, L. Zhang, Y. Zhao, D. K. St Clair and I. Batinić-Haberle, "Mn Porphyrin-Based SOD Mimic, MnTE-2-PyP ${ }^{5+}$ Targets Mouse Heart Mitochondria," Free Radical Biology and Medicine, Vol. 42, No. 8, 2007, pp. 1193-1200. doi:10.1016/j.freeradbiomed.2007.01.019

[24] I. Spasojevic, Y. Chen, T. J. Noel, P. Fan, L. Zhang, J. S. Rebouças, D. K. St Clair and I. Batinić-Haberle, "Pharmacokinetics of the Potent Redox-Modulating Manganese Porphyrin, MnTE-2-PyP ${ }^{5+}$, in Plasma and Major Organs of B6C3F1 Mice," Free Radical Biology and Medicine, 
Vol. 45, No. 7, 2008, pp. 943-949. doi:10.1016/j.freeradbiomed.2008.05.015

[25] B. J. Day, S. Shawen, S. I. Liochev and J. D. Crapo, "A Metalloporphyrin Superoxide Dismutase Mimetic Protects against Paraquat-Induced Endothelial Cell Injury, in Vitro," The Journal of Pharmacology and Experimental Therapeutics, Vol. 275, No. 3, 1995, pp. 1227-1232.

[26] M. Patel, B. J. Day, J. D. Crapo, I. Fridovich and J. O. McNamara, "Requirement for Superoxide in Excitotoxic Cell Death," Neuron, Vol. 16, No. 2, 1996, pp. 345-355. doi:10.1016/S0896-6273(00)80052-5

[27] B. J. Day and J. D. Crapo, "A Metalloporphyrin Superoxide Dismutase Mimetic Protect against Paraquat-Induced Lung Injury in Vivo," Toxicology and Applied Pharmacology, Vol. 140, No. 1, 1996, pp. 94-100. doi:10.1006/taap.1996.0201

[28] B. J. Day, I. Fridovich and J. D. Crapo, "Manganic Porphyrins Possess Catalase Activity and Protect Endothelial Cells against Hydrogen Peroxide-Mediated Injury," Archives Biochemistry Biophysics, Vol. 347, No. 2, 1997 , pp. 256-262. doi:10.1006/abbi.1997.0341

[29] J. Milano and B. J. Day, "A Catalytic Antioxidant Metalloporphyrin Blocks Hydrogen Peroxide-Induced Mitochondrial DNA Damage," Nucleic Acids Research, Vol. 28, No. 4, 2000, pp. 968-973. doi:10.1093/nar/28.4.968

[30] C. Szabó, B. J. Day and A. L. Salzman, "Evaluation of the Relative Contribution of Nitric Oxide and Peroxynitrite to the Suppression of Mitochondrial Respiration in Immunostimulated Macrophages Using a Manganese Mesoporphyrin Superoxide Dismutase Mimetic and Peroxynitrite Scavenger," FEBS Letters, Vol. 381, No. 1-2, 1996, pp. 82-86. doi:10.1016/0014-5793(96)00087-7

[31] G. Ferrer-Sueta, L. Ruiz-Ramirez and R. Radi, "Ternary Copper Complexes and Manganese(III) Tetrakis(4-benzoic acid)porphyrin Catalyze Peroxynitrite-Dependent Nitration of Aromatics," Chemical Research in Toxicology, Vol. 10, No. 12, 1997, pp. 1338-1344. doi:10.1021/tx970116h

[32] I. L. Jackson, L. Chen, I. Batinić-Haberle and Z. Vujaskovic, "Superoxide Dismutase Mimetic Reduces Hypoxia Induced-O ${ }^{2-}$, TGF- $\beta$ and VEGF Production by Macrophages," Free Radical Research, Vol. 41, No. 1, 2007, pp. 8-14. doi:10.1080/10715760600913150

[33] B. Gauter-Fleckenstein, K. Fleckenstein, K. Owzar, C. Jian, J. S. Rebouças, I. Batinić-Haberle and Z. Vujaskovic, "Early and Late Administration of MnTE-2-PyP ${ }^{5+}$ in Mitigation and Treatment of Radiation-Induced Lung Damage," Free Radical Biology and Medicine, Vol. 48, No. 8, 2010, pp. 1034-1043. doi:10.1016/j.freeradbiomed.2010.01.020

[34] L. Y. Chang and J. D. Crapo, "Inhibition of Airway Inflammation and Hyperreactivity by a Catalytic Antioxidant," Chest, Vol. 12, No. 3, 2003, p. 446S.
[35] L. Y. Chang and J. D. Crapo, "Inhibition of Airway Inflammation and Hyperreactivity by an Antioxidant Mimetic," Free Radical Biology and Medicine, Vol. 33, No. 3, 2002, pp. 379-386. doi:10.1016/S0891-5849(02)00919-X

[36] L.-Y. Chang, M. Subramaniam, B. A. Yoder, B. J. Day, M. C. Ellison, M. E. Sunday and J. D. Crapo, "A Catalytic Antioxidant Attenuates Alveolar Structural Remodeling in Bronchopulmonary Dysplasia," American Journal of Respiratory and Critical Care Medicine, Vol. 167, No. 1, 2003, pp. 57-64. doi:10.1164/rccm.200203-232OC

[37] H. M. Tse, M. J. Milton and J. D. Piganelli, "Mechanistic Analysis of the Immunomodulatory Effects of a Catalytic Antioxidant on Antigen-Presenting Cells: Implication for Their Use in Targeting Oxidation-Reduction Reactions in Innate Immunity," Free Radical Biology and Medicine, Vol. 36, No. 2, 2004, pp. 233-247. doi:10.1016/j.freeradbiomed.2003.10.029

[38] J. D. Piganelli, S. C. Flores, C. Cruz, J. Koepp, I. Batinić-Haberle, J. Crapo, B. Day, R. Kachadourian, R. Young, B. Bradley and K. Haskins, “A MetalloporphyrinBased Superoxide Dismutase Mimic Inhibits Adoptive Transfer of Autoimmune Diabetes by a Diabetogenic TCell Clone," Diabetes, Vol. 51, No. 2, 2002, pp. 347- 355. doi:10.2337/diabetes.51.2.347

[39] A. Y. Makinde, A. Rizvi, J. D. Crapo, R. D. Pearlstein, J. M. Slater and D. S. Gridley, "A Metalloporphyrin Antioxidant Alters Cytokine Responses after Irradiation in a Prostate Tumor Model," Radiation Research, Vol. 173, No. 4, 2010, pp. 441-452. doi:10.1667/RR1765.1

[40] X. W. Mao, J. D. Crapo, T. Mekonnen, N. Lindsey, P. Martinez, D. S. Gridley and J. M. Slater, "Radioprotective Effect of a Metalloporphyrin Compound in Rat Eye Model," Current Eye Research, Vol. 34, No. 1, 2009, pp. 62-72. doi: $10.1080 / 02713680802546948$

[41] T.-J. Wu, N. H. Khoo, F. Zhou, B. J. Day and D. A. Parks, "Decreased Hepatic Ischemia-Reperfusion Injury by Manganese-Porphyrin Complexes," Free Radical Research, Vol. 41, No. 2, 2007, pp. 127-134. doi: $10.1080 / 10715760600801298$

[42] G. B. Mackensen, M. Patel, H. Sheng, C. L. Calvi, I. Batinić-Haberle, B. J. Day, L. P. Liang, I. Fridovich, J. D. Crapo, R. D. Pearlstein and D. S. Warner, "Neuroprotection from Delayed Postischemic Administration of a Metalloporphyrin Catalytic Antioxidant," Journal of Neuroscience, Vol. 21, No. 13, 2001, pp. 4582-4592.

[43] H. Yao, G. Arunachalam, J.-W. Hwang, S. Chung, I. K. Sundar, V. L. Kinnula, J. D. Crapo and I. Rahman, "Extracellular Superoxide Dismutase Protects against Pulmonary Emphysema by Attenuating Oxidative Fragmentation of ECM," Proceedings of the National Academy of Sciences of USA, Vol. 107, No. 35, 2010, pp. 1557115576. doi:10.1073/pnas. 1007625107 